\title{
Manual therapy for chronic migraine: a pragmatic randomised controlled trial study protocol
}

\author{
Jim Odell ${ }^{1 *}$ (D), Carol Clark ${ }^{1}$, Adrian Hunnisett ${ }^{2}$, Osman Hassan Ahmed ${ }^{1}$ and Jonathan Branney ${ }^{1}$
}

\begin{abstract}
Introduction: Chronic migraine is a largely refractory condition affecting between 1 and 2.2\% of the overall population worldwide, with females more affected than males. There are also high health and socioeconomic costs associated both for the individual and society. The mainstay of chronic migraine management is pharmacological, but the options available have limited efficacy and there are often unwanted side effects. There is some evidence for manual therapy as a treatment option for migraine, but its effectiveness for chronic migraine is unknown. Therefore, we have designed a pragmatic randomised control trial to investigate whether adding manual therapy to the tertiary specialist treatment of chronic migraine improves patient-reported outcomes.
\end{abstract}

Methods: A pragmatic, randomised controlled trial in a hospital tertiary headache clinic. Participants will be randomised into one of two groups: treatment as usual or treatment as usual plus manual therapy. The primary outcome measure will be a change in the Headache Impact Test score. Secondary outcomes will also be measured over the 12-week study period including changes in headache frequency, migraine specific quality of life and reductions in relevant medicine consumption. The manual therapy group will have five treatment sessions each lasting $30 \mathrm{~min}$. The recruitment target of 64 participants will allow power at $80 \%$ with $p=0.05$ using minimal clinical difference for Headache Impact Test of 3.7 and includes provision for a $10 \%$ dropout rate. Recruitment will take place between August 2018 and February 2019. The results will form part of a doctoral study and be published in peer-reviewed journals and presented at national/international conferences.

Discussion: Current pharmacological approaches have limited effects in the management of chronic migraine and there is a requirement to improve treatment options and reduce the health and economic burden of the condition. Manual therapy has been shown to be effective in other chronic pain conditions as well as other primary headaches. This study will explore the effectiveness of manual therapy as an adjunctive approach to the management of chronic migraine.

Trial registration: The trial has received a favourable opinion from the UK Health Research Authority (IRAS 228901) and is registered at ClinicalTrials.gov.number NCT03395457. Registered 1st March 2018.

Keywords: Chiropractic, Chronic migraine, Headache, Manual therapy, Allodynia, Randomised controlled trial

\footnotetext{
* Correspondence: jodell@bournemouth.ac.uk

'Bournemouth University, Royal London House, Christchurch Road,

Bournemouth BH1 3LT, UK

Full list of author information is available at the end of the article
}

(c) The Author(s). 2019 Open Access This article is distributed under the terms of the Creative Commons Attribution 4.0 International License (http://creativecommons.org/licenses/by/4.0/), which permits unrestricted use, distribution, and reproduction in any medium, provided you give appropriate credit to the original author(s) and the source, provide a link to the Creative Commons license, and indicate if changes were made. The Creative Commons Public Domain Dedication waiver (http://creativecommons.org/publicdomain/zero/1.0/) applies to the data made available in this article, unless otherwise stated. 


\section{Background}

Migraine is experienced by the vast majority of sufferers as episodic migraine (EM) $[1,2]$ occurring regularly although not necessarily frequently, with around $65 \%$ of sufferers estimated to have a migraine episode fortnightly to monthly [3]. However if the episodic pattern becomes uncontrolled, a process of chronification occurs whereby the original episodic migraine becomes very frequent and more disabling. This is termed chronic migraine (CM) and is described by the International Headache Society (IHS) classifications [4] as "headache occurring on 15 or more days per month for more than three months, which, on at least 8 days per month, has the features of migraine headache". On the days without migraine headache the individual can often suffer from pre and post headache effects adding to the burden of this condition.

The management of CM is more complex than EM, as it is often resistant to standard treatments with resultant additional costs compared to EM. The mean (SD) annual cost per CM person $(\$ 8243[\$ 10,646])$ was over three times that of those with EM (\$2649 [\$4634]). Both direct medical costs and cost of lost productivity were substantially higher in CM than EM [5]. In addition to financial costs, the social costs and personal impact of $\mathrm{CM}$ often leads to severe disability for those with $\mathrm{CM}[6$, 7]. This is especially true for females, with the annual prevalence of CM in women being 1.7-4.0\% compared to men $(0.6-0.7 \%)$ [8-11]. Those women aged between 18 and 49 years of age are the most affected across the range of measures. In one study, $\mathrm{CM}$ sufferers were found to be three times more likely to have lost work and have reduced household productivity than those with EM (58\% compared to 18\%) [12]. People with CM are also much more likely to report "very severe headache-related disability" as measured by the Migraine Disability Assessment Scale (MIDAS) than those with episodic (24.8 and 3.2\% respectively) [13, 14].

Despite the suggestion that migraine is a syndrome with multiple pathological mechanisms which support a multi therapeutic approach rather than a single approach [15-17], the mainstay of treatment for $\mathrm{CM}$ is pharmacological. However, some patients do not want, or cannot, take some prophylactic medications such as Topiramate due to restrictions in its use [18]. Currently, OnabotulinumtoxinA (Botox) is the only specifically licensed treatment for CM in the UK [19]. Although its mechanism of action is unclear, studies have demonstrated that injecting specific sites on the head and neck produce significantly beneficial effects in $\mathrm{CM}$ patients. One study concluded that $32 \%$ of CM patients achieved a $50 \%$ reduction in headache days and a $50 \%$ reduction in migraine days, with NICE guidance recommending a $30 \%$ reduction in headache days as a measure of success in Botox studies [20]. Although similar clinically significant reductions in the Headache Impact Test (HIT 6) scores are seen with Botox and Topiramate interventions, Botox has fewer side effects and higher adherence rates $[21,22]$. However, the efficacy of Botox in those patients who benefit only partially or not at all from Botox, and its relatively high cost, are barriers to an increased uptake [23-25]. Therefore if adjunctive therapies, especially those with fewer side effects and relatively low costs could be utilised, this may increase the benefit to more of those with CM.

One possible adjunctive intervention is manual therapy. Despite the mechanisms of its potential action in $\mathrm{CM}$ being unknown, the basis for its potential role can be garnered from studies in associated conditions. These include primary headaches (tension type and migraine), as well as common comorbidities; chronic pain and specifically neck pain. One systematic review concluded that MT has an efficacy in the treatment of chronic tension headache equal to that of prophylactic medication with tricyclic antidepressant [26]. Another involving massage therapy and chiropractic spinal manipulative therapy concluded that they may be as efficient as Propranolol and Topiramate in migraine prophylaxis. It also concluded that there is moderate quality evidence for both spinal manipulation and mobilisation as suitable treatments in chronic non-specific neck pain [27]. These studies found benefits in at least one of the following: frequency, duration and intensity of headaches/pain.

Currently there are two theories in existence relevant to this study of manual therapy in the management of chronic migraine. One is that migraine is, in part, an abnormal response in those genetically pre-disposed, to nociceptive input involving the nerves of the upper cervical spine $(\mathrm{C} 1, \mathrm{C} 2$ and $\mathrm{C} 3)$ and associated joints and muscles. This leads to exaggerated sensitisation of the trigeminal pathway and subsequent face, neck and head pain [28-30].

The other theory incorporates the allostatic model in which people respond to stressful events, actual or perceived, with physiological and behavioural changes. In general these changes maintain normal physiological stability (allostasis). However, if stressors (including ongoing pain or nociceptive inputs) become too great or frequent then the normal response can become dysfunctional as a result of allostatic loading, which itself alters brain structure and function. Likewise, repeated migraines are themselves thought to be a driver of changes to the brain structure that may lead to a dysfunctional allostatic response. Consequently, many migraine sufferers report that stressful activities of daily living (physical and emotional) exacerbate their migraines [31, 32].

Chronification from EM to CM may be a result of the stress mechanisms generating the migraine or as a 
consequence of changes in the brain arising in response to the attacks. Therefore if stressors (for example nociceptive pain, and central sensitisation, possibly from musculoskeletal disorders) can be reduced, this may add to the efficacy of existing interventions [33].

Whilst the process of chronification in CM is not fully understood, there are a number of known associated risk factors including head and neck injury, and other pain disorders [34] (Table 1).

Currently, one of the most common factors in chronification is considered to be the presence of medication over use headaches $(\mathrm{MoH})$ due to the frequent use of opioids and barbituates in self medication of headaches, or in association with concomitant conditions. Estimates of between 30 and $72 \%$ of CM patients presenting at tertiary clinics are thought to have $\mathrm{MoH}$ associated chronification $[35,36]$. The IHS diagnosis of medication overuse is made if abortive drugs are used regularly for more than three months on 10 or more, or 15 or more, days a month, depending on the drug. One study estimated a twofold increase in chronification with opioid use on 8 days a month [37]. Therefore any studies of interventions in $\mathrm{CM}$ should include a detoxification phase or a process to mitigate $\mathrm{CM}$ with $\mathrm{MoH}$ being included.

Both of the above migraine models suggest a role for altered sensory processing in the brainstem, which is associated with the presence of central sensitisation and one of the consequences, cutaneous allodynia (CA). Some studies cite CA as more prevalent in $\mathrm{CM}$ than $\mathrm{EM}$, whilst others suggest there is little difference and it is more a reflection of migraine duration. There are a few potential reasons for these differences which include: the type of CA - thermal, dynamic and static mechanical; how it is measured and the temporal nature of CA making it difficult to measure consistently; and its role

Table 1 Risk factors associated with migraine chronification and reversion

- Obesity

- Snoring

- Sleep disorders

- Excessive caffeine intake

- Psychiatric/psychological disease (Depression/Anxiety)

- High baseline headache frequency

- Overuse of migraine abortive drugs

- Major life changes

- Head or neck injury

- Cutaneous allodynia

- Female sex

- Comorbid pain disorders

- Lower socioeconomic status

(Adapted from Schwedt, 2014, [79]) as a marker for the risk of frequent attacks rather than simply a consequence of frequent attacks [38, 39]. CA is also consistently reported more in females than males (49.7\% vs $32.6 \% \%, P<0.001)$ [40] and is common in other chronic pain conditions, with its presence associated with a reduction in the efficacy of abortive treatments $[41,42]$.

Various models of MT and pain reduction exist which involve biomechanical, neurophysiological and psychological components, either individually or in combination. However all of these models consider that MT may work by activating descending inhibitory pathways via different levels of the spinal cord [43-45]. The common relationship of central sensitisation, the cervical spine, and pain disorders (see Table 1) would suggest MT may have utility in the management of CM.

In terms of potential as an adjunctive treatment, MT has been shown to reduce pain and have a direct effect on the mechanics of the cervical spine that results in functional improvements [46-48]. MT has also been shown to increase local pain pressure thresholds, which are used to detect central sensitisation $[49,50]$. Consequently, MT may reduce cutaneous allodynia and improve the efficacy of current approaches to treating CM.

The above discussion outlines commonalities between migraine, other pain conditions, its chronification and the potential for MT in its treatment. Combined with evidence showing the scope for improvement in existing $\mathrm{CM}$ treatment, this suggests there may be potential for the use of adjunctive non-pharmacological treatments, especially if these are generally associated with lesser side effects $[16,51]$.

The objective of this study is to determine the effectiveness of MT as an adjunctive therapy to tertiary care ('care as usual)' in CM.

\section{Methods \\ Study design}

This will be a single centre, pragmatic Randomised Controlled Trial (RCT) involving 64 participants. The two groups will be "Tertiary care as usual" and "Tertiary care as usual plus MT". The objective of the study is to measure the difference between the two treatment groups in $\mathrm{CM}$. The study design adheres to the IHS and Consolidated Standards of Reporting Trials (CONSORT) guidelines $[52,53]$. The study flow is outlined in Fig. 1.

\section{Participants \\ Recruitment}

Participants will be recruited between August 2018 and February 2019 through the Salford Royal NHS Foundation Trust acute neurology clinic service in the UK. The neurologist and specialist headache nurse will identify 


\section{Chronic migraine patients identified in Pre-Screen by headache clinic}

\section{Contact Existing Chronic Migraine Patients by letter}

$\sim 4$ weeks before next appointment. A patient information sheet will be provided and instructions for following up.

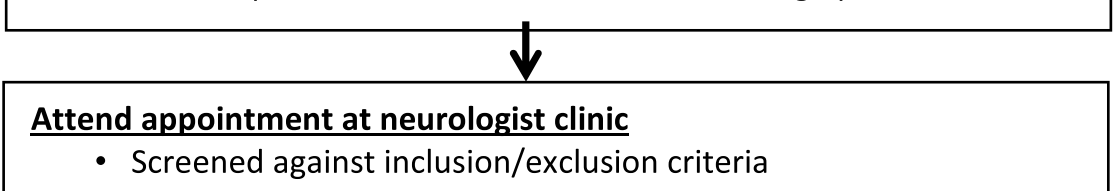

- Screened against inclusion/exclusion criteria

Initial meeting with PI on same day as neurologist appointment or arrangement made for alternative day within next week

- Informed consent obtained for study

- $\quad$ Randomised into MT or CAU group (neurologist/nurse is blinded)

- Baseline survey data confirmed

- $\quad$ Algometry and BMI measurements taken for both groups

- Migraine diary explained and implemented

- Treatment schedule arranged
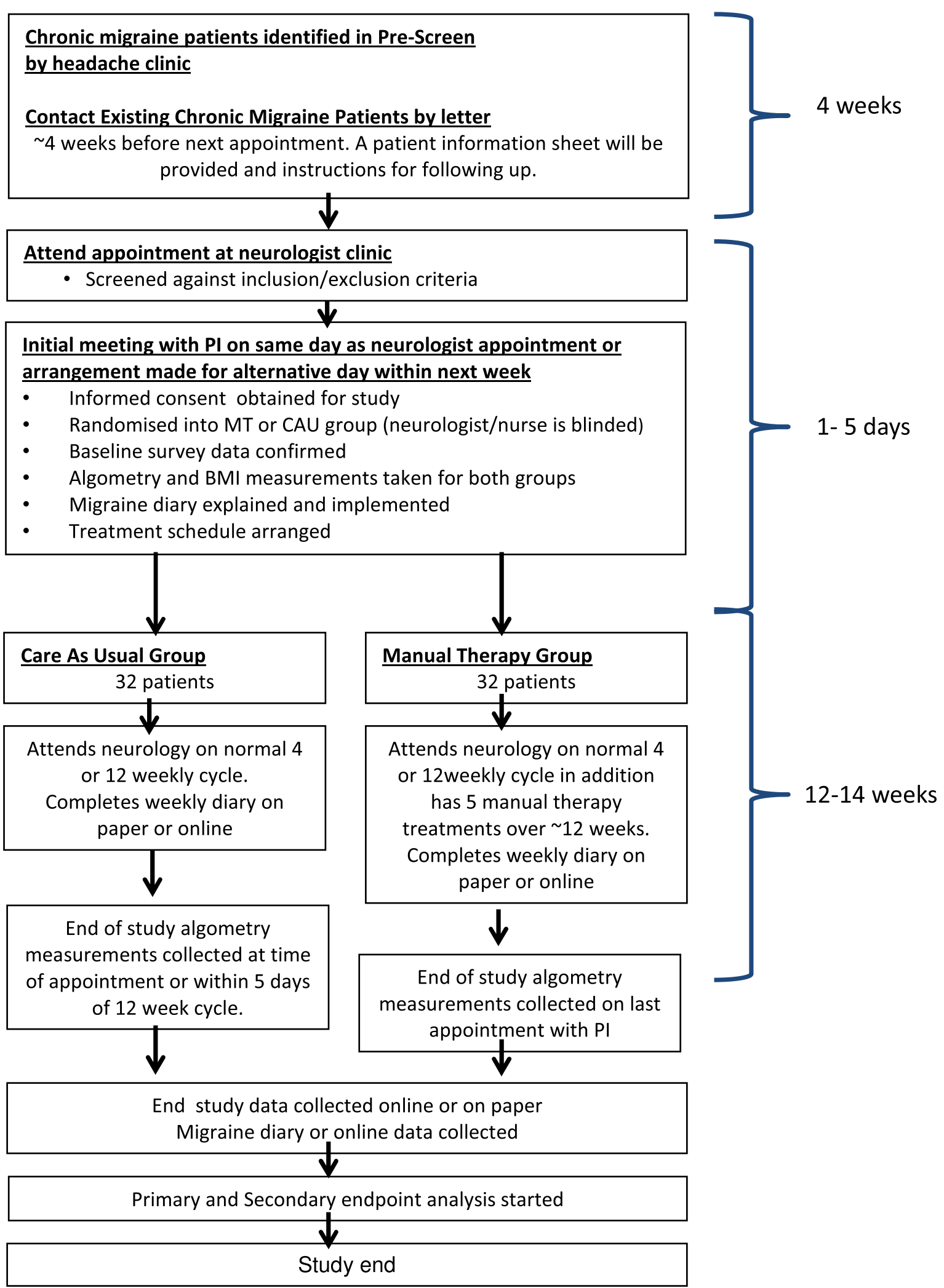

Fig. 1 Participant flow 
potential participants from their list of existing $\mathrm{CM}$ patients.

Details of this study will be sent to potential participants with their clinic appointment letter. Eligible participants will be female aged greater than 18 years of age, and diagnosed with $\mathrm{CM}$ according to the criteria of the International Classification of Headache Disorders (ICHD-III) by their neurologist. The enrolment in to tertiary care requires that patients must be free of medication over use headache and as part of the inclusion criteria (Table 2), participants will have gone through a detoxification phase and had at least one cycle of tertiary care treatment to further mitigate medication overuse headache. Exclusion criteria for this study are: contraindication to MT including spinal radiculopathy; uncontrolled psychological conditions; and any physical or MT in the last 6 weeks. Acute and abortive migraine medication will continue as usual during the study.

\section{Inclusion and exclusion criteria Sample size}

The study is powered at $80 \%(\alpha=0.05)$ to detect a mean difference of 3.7 points in HIT6 before and after treatment. A pooled calculation of standard deviation (SD) of 4.91 was calculated for the Headache Impact Test

Table 2 Inclusion and exclusion criteria

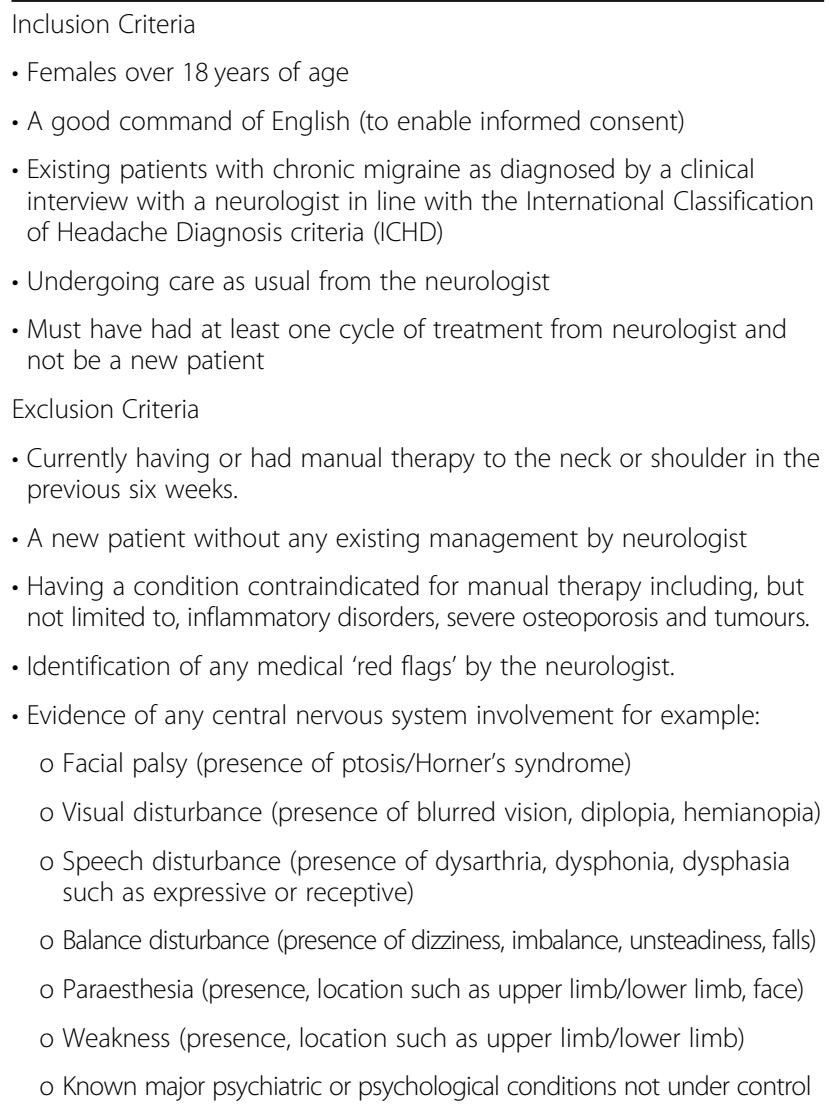

(HIT6) from the analysis of major studies in chronic migraine [7, 54-59]. Although this requires 29 participants in each group, 32 will be recruited to allow for attrition that may be experienced over a 12 week period.

\section{Allocation}

A randomisation assignment sequence will be created by an independent member of the research team using randomisation software "Research Randomizer" [60]. The results will be put into sealed opaque envelopes which the participants will open in their meeting with the PI, who will explain the process to be followed depending on the group allocation.

Immediately following the initial screening by the neurologist/specialist nurse, those participants who meet the inclusion criteria will be invited to an assessment with the Principal Investigator (PI). If this is not possible, it will be arranged within 5 days. The PI will provide participants with verbal and written information about the project before gaining written consent. He will also explain the risks, and benefits as well as the potential adverse reactions to MT and how to manage them. Potential adverse reactions may include temporary local tenderness, aching and tiredness [61]. Participants will be randomised into two groups (care as usual and care as usual plus MT).

The neurologist will be blinded as to the participant's group allocation in order to reduce initial selection and potential contact bias. The PI cannot be blind to the manual therapy group but will be blinded to the end of study survey analysis, with an independent member of the research team detailed to recode the participant reference numbers and store the key file on a password protected computer. The unblinding of single cases by the $\mathrm{PI} / \mathrm{CI}$ in the course of a clinical trial will only be performed if necessary for the safety of the trial subject.

\section{Intervention}

The neurologist will manage the care as usual group alone. The care as usual and MT group will be managed by the neurologist and receive MT from the PI. The MT administered will consist solely of mobilisation, manipulation and soft tissue work. The PI will use a pragmatic approach to the MT administered, using any of the above manual interventions deemed clinically appropriate at each appointment based on assessment prior to treatment and participant feedback. The PI is an experienced chiropractor with over ten years' clinical experience of chiropractic and soft tissue work, with postgraduate education and training in the management of CM. The treatment delivered will be recorded in the Case Report File (CRF). Failure to attend all manual therapy sessions will result in withdrawal from the study although provision will be made to provide an alternative 
appointment if the participant cannot make one as planned. Any change in a participant's medical condition such that they fulfil the exclusion criteria above will result in their withdrawal from the study. Data from these participants would not be included in the final study results. Adverse events will be recorded in the CRF, and if classed as serious would be referred to the neurologist to be reported to the sponsor within $24 \mathrm{~h}$ of learning of the event and to the Main Regional Ethics Committee within 15 days in line with the required timeframe. The outline interventional procedure is shown in Table 3 with the project flow following the SPIRIT guidelines (Table 4).

\section{Outcome measures}

The primary and secondary endpoints are differences in within-group changes between groups in the outcomes listed below (Table 5). These will be measured using the weekly diaries and the validated instruments in the baseline and 12-week follow-up questionnaires.

\section{Data collection}

All participants will be asked to complete a baseline questionnaire, comprising demographic information and the following validated instruments: Headache Impact Test (HIT6), the Migraine Specific Quality of Life Questionnaire (MSQ.V2),The perceived Stress Scale (PSS-10), State and Trait Anxiety Inventory (STAI-6),Brief Cope, Hospital Anxiety and Depression Scales (HADS) and the 12 Item Allodynia Symptom Checklist (ASC-12) The questionnaire will be available in paper or online format for completion at the initial assessment or at home at the discretion of the participant.

BMI values (from height and weight) will be calculated in the clinic and pain pressure thresholds will be taken using a somedic electronic pressure algometer [62] on each of the following muscles: Frontalis, Trapezius and Subocciptal. The order of these measurements will be

\section{Table 3 Manual therapy protocol}

\footnotetext{
1. Assess upper body ${ }^{a}$ posture in sitting

2. Assess active and passive neck range of motion

3. Assess shoulder girdle range of motion by raising each arm sideways from side of body up to ear

4. Assess the temporomandibular joint

5. Identify areas to treat in sitting position

6. Administer MT using mobilisation, manipulation and soft tissue release in sitting position

7. Assess patient shoulder girdle, neck and head supine and prone

8. Administer MT in supine and prone position

9. Following each session an outline of the MT used will be recorded.

10. A total of 30 min will be allocated for each participant at these consultations
}

apper body defined as from thoraco-lumbar junction upwards taken randomly, which is an approach used in previous migraine studies [47, 48]. A distal site (forearm) will also be included to help provide a measure of wide spread central sensitisation [63]. These measures will be taken again at the end of the study after 12 weeks. Individuals assigned to the tertiary care as usual plus MT group will be given a 12 week treatment plan as recommended in IHS guidelines [52]. Sessions will be planned for Weeks $1,2,5,8$ and 12.The care as usual group will complete the normal 12 week cycle with the neurologist. Both groups will complete weekly diaries and an end of study questionnaire that duplicates the baseline questionnaire and includes a Patient Global Impression of Change Scale.

To encourage completion of data the participants will be encouraged to complete paper versions of the questionnaire at the first visit in the waiting area and leave it at reception, rather than taking home. Online versions will also be available. Reminder text message or emails will be sent out each week to encourage completion of the paper or online weekly diary.

\section{Data management}

The questionnaires and diaries will not contain information that can identify individuals, only an individual reference number. All information used for this research will be kept securely on password protected computers and only accessible by members of the research team. Any data that can identify a participant will be destroyed within 3 months of final data collection. Anonymous data may be kept for up to 5 years on a secure university computer.

\section{Statistical analysis}

Descriptive demographic and clinical characteristics will be collected, using means and SDs for continuous variables and proportions and percentages for nominal variables. Each group will be described separately. Quantitative data collected from each group will be analysed using SPSS ${ }^{(\mathrm{m})}$ Version 24 software. Parametric tests will be used to analyse data wherever it is normally distributed, and non-parametric will be utilised for non-normal data.

The mean and standard deviations of primary and secondary outcomes will be reported, with an analysis of between- group differences over the 12 weeks using Students T test or Mann-Whitney. The effect size will be calculated using the following figures: small $(0.2-0.5)$, medium $(0.5-0.8)$ or large $(>0.8)$. Repeated measures ANOVA will be used to explore the differences between intermediate time points. If appropriate, multivariate linear regression (MANOVA) will assess the secondary outcome relationship with changes in primary outcomes. 
Table 4 Project Flow Manual Therapy for Chronic Migraine

\begin{tabular}{|c|c|c|c|c|c|c|c|}
\hline \multirow[b]{3}{*}{ TIMEPOINT ${ }^{\star *}$} & \multicolumn{7}{|c|}{ STUDY PERIOD } \\
\hline & \multirow{2}{*}{$\begin{array}{c}\text { Enrolment } \\
-2 w\end{array}$} & \multirow{2}{*}{$\begin{array}{c}\text { Allocation } \\
0\end{array}$} & \multicolumn{4}{|c|}{ Post-allocation } & \multirow{2}{*}{$\frac{\text { Close-out }}{+12 w}$} \\
\hline & & & $+1 w$ & $+2 w$ & $+5 w$ & $+8 w$ & \\
\hline \multicolumn{8}{|l|}{ ENROLMENT: } \\
\hline \multirow{2}{*}{$\begin{array}{l}\text { Eligibility screen } \\
\text { Informed consent }\end{array}$} & $x$ & & & & & & \\
\hline & & $x$ & & & & & \\
\hline Allocation & & $x$ & & & & & \\
\hline \\
\hline \multicolumn{8}{|l|}{$\begin{array}{l}\text { Group } 1 \\
\text { Neurological Care as } \\
\text { Usual }\end{array}$} \\
\hline $\begin{array}{c}\text { Group } 2 \\
\text { Manual Therapy Input } \\
\text { to Neurological care } \\
\text { as Usual }\end{array}$ & & & $x$ & $x$ & $x$ & $x$ & $x$ \\
\hline \multicolumn{8}{|l|}{ ASSESSMENTS: } \\
\hline \multirow{4}{*}{$\begin{array}{l}\text { Investigator } \\
\text { Assessed Measures: } \\
\text { Pain pressure threshold } \\
\text { BMI } \\
\text { Self Report } \\
\text { Measures: } \\
\text { Perceived Stress Scale } \\
\text { State and Trait Anxiety } \\
\text { Inventory } \\
\text { Headache Impact Test; } \\
\text { Migraine Specific Quality } \\
\text { of Life; } \\
\text { Brief Cope } \\
\text { Hospital Anxiety and } \\
\text { Depression Scale) } \\
\text { Headache Diary } \\
\text { Perceived Stress } \\
\text { Number headaches } \\
\text { Intensity headaches } \\
\text { Sleep quality } \\
\text { Medication usage } \\
\text { Diet quality } \\
\text { Triggers } \\
\text { Allodynia Symptom } \\
\text { Checklist }\end{array}$} & & $x$ & & & & & $X$ \\
\hline & & $x$ & & & & & $x$ \\
\hline & & & & & & & \\
\hline & & & & & & & \\
\hline
\end{tabular}

Correlation between primary and secondary outcomes will use Pearson's coefficient.

\section{Discussion}

Two high quality RCTs have explored the effectiveness of MT in those with migraine, of which only one study specifically reported on those with a diagnosis of CM $[64,65]$. This was a 3-Armed RCT with 105 participants using osteopathy (MT) and medical therapy, sham and medical therapy and medical therapy alone. The results were a reduction in HIT-6 score by an average of- 8.74; $p<0.001$ with OMT compared to medication care as 
Table 5 Outcome Measures

\begin{tabular}{ll}
\hline Primary Outcomes & Measurement Instrument (validity) \\
Migraine-related disability & Headache Impact Test (HIT 6) [54] \\
Secondary Outcomes & Measurement Instrument \\
Quality of Life & $\begin{array}{l}\text { Migraine Specific Quality of Life } \\
\text { Questionnaire (MSQ. V2.1)[59] }\end{array}$ \\
Use of abortive migraine medications & 1.1.11.1.11.1.1Diary [69] \\
Percentage of participants with & \\
reduction in headache frequency \\
(days per month) of greater than 50\%
\end{tabular}

usual. There were also statistically significant $(p<0.001)$ reductions in medication use and migraine frequency. In comparison, this study will use only one practitioner instead of six, and five sessions over three months instead of eight sessions over six months. The technique used will be chiropractic rather than osteopathy, although similar soft tissue release and mobilisation techniques will be used (albeit on a broader area of the shoulder girdle and neck). This difference with a crossover of similarity should strengthen the theory that manual therapy of different types can produce similar adjunctive benefits and add to the dose benefit discussion.

There is evidence to suggest that $\mathrm{MT}$ as an adjunct to Botox improves outcomes in those with CM. This comes from a pilot study with 22 participants [66]; one group had transcutaneous electrical nerve stimulation (TENS) and Botox, the other MT and Botox. Each group had one 30 min session per week over 4 weeks. Both groups showed a reduction in medication use and pain pressure thresholds in the MT group compared to the TENS. This study aims to build on the outcomes of previous studies focussing on MT as an adjunctive therapy to 'usual care' for those receiving treatment in a tertiary care setting. At the time of writing, this study is thought to be the first to measure central sensitisation using algometry in CM whilst tracking changes using the Allodynia Symptom Checklist. This will allow greater understanding of potential temporal changes in central sensitisation with treatment outcomes. It should also provide a comparison between the baseline and final algometry changes and the Allodynia Symptom Checklist measures.

\section{Methodological considerations}

The study is designed to be pragmatic and to balance the needs of efficacy and effectiveness in clinical research [67]. The HIT 6 score is used in this study as the primary outcome measure, as this is the only headache disability measurement tool with established validity in $\mathrm{CM}[54,57]$. The number of headache/migraine days will be collected as a secondary measure using a headache diary, a well established and valid measurement instrument [68-70] and recommended by the IHS.

Blinding and the placebo effects are potential limitations in this study. Although it is impossible to blind the PI for treatment, the final results will be re-coded to reduce the potential for analysis bias. The placebo effect is known to be high in all headache studies with acute treatment placebo being higher than prophylactic and tablets lower than injection [71, 72]. In studies of botox in chronic migraine, both in the immediate 12 week cycle and in longer term studies over multiple cycles, authors have cited placebo levels of between 20 and $49 \%[73,74]$.

It is recognised that the MT group may experience a higher level of placebo as a result of the necessary interpersonal interactions $[75,76]$. However in this study there is a high level of personal interaction with the headache nurse who applies the medical interventions and thus may be considered a balancing to interactions in the MT sessions.

The external validity of the RCT may be a weakened as a result of the involvement of only one manual therapist, and thus could limit the measure of consistency of outcomes across different practitioners. Conversely, this will reduce the risk of potential multiple bias that comes with the use of multiple therapists. To mitigate reductions in external validity, the study focuses on a specific group in a 'real life setting' (females aged 18 years and above with $\mathrm{CM}$ in tertiary clinics) [77]. It will measure factors including stress, depression, coping styles, anxiety, diet, sleep quality, and allodynia, all of which potentially affect the primary and secondary outcomes. Although the IHS recommend a placebo control for prophylactic pharmacological studies, there is evidence that less than $10 \%$ actually conform [78] and likewise despite having two active arms this study also lacks a placebo control group. To address this limitation, and in the absence of IHS guidelines for non-pharmacological trials, the study design will adhere closely to the recommendations for IHS pharmacological RCTs in chronic migraine. The CONSORT guideline for non-pharmacological studies will be followed which provides a systematic 
approach to ensuring quality and the external and internal validity of clinical studies.

\section{Scientific value}

$\mathrm{CM}$ is a highly disabling condition with few evidencebased treatment options. In the UK, Botox is the only specifically approved pharmacological intervention, although Topiramate and occipital nerve injections are used in tertiary clinics. This study will aim to explore the outcomes of MT as an adjunct to those receiving 'care as usual' for $\mathrm{CM}$ in a tertiary setting. It will also provide information relating to central sensitisation in the form of CA for participants, which will contribute to a body of knowledge in those with $\mathrm{CM}$ and support tailoring treatment options.

Although this will be a pragmatic study, the results of this study will only be applicable to the target group - females over 18 years of age with $\mathrm{CM}$ under tertiary care. The approach used will however be generalisable to all manual therapists with the requisite training in techniques used. Undertaking the study in a specialist medical headache clinic should help to build the nascent relationship between medical headache specialists and manual therapists. Post study dissemination will potentially help the development of more multidisciplinary approaches.

\begin{abstract}
Abbreviations
ASC: Allodynia Symptom Checklist; CA: Cutaneous Allodynia; CM: Chronic Migraine; CRF: Case Report File; EM: Episodic Migraine; HADS: Hospital Anxiety and Depression Scale; HIT6: Headache Impact Test; IHS: International Headache Society; MIDAS: Migraine Disability Assessment Scale; MoH: Medication Overuse Headache; MSQ. V2.1: Migraine Specific Quality of Life Questionnaire; MT: Manual Therapy; PGICS: Patient Global Impression of Change Scale; PI: Principal Investigator; PSS: Perceived Stress Questionnaire; STAI-6: State and Trait Anxiety Inventory
\end{abstract}

\section{Acknowledgments}

None

\section{Funding}

This is part of a PhD and is funded by Bournemouth University (BU), the Royal College of Chiropractors (RCC) and the McTimoney College of Chiropractors (MCC).

\section{Availability of data and materials}

Data sharing is not applicable to this article as no datasets were generated or analysed during the current study.

\section{Dissemination}

This project is due for completion in September 2019 as part of the submission for PhD. The results (positive, negative or inconclusive) will be published in peer-reviewed, international scientific journals. It will be disseminated via posters and/or oral presentations at national and international conferences, and summaries will be provided for migraine charities and associated organisations.

\section{Sponsor contact details}

Suzy Wignall

Clinical Governance Advisor

Research and Knowledge Exchange Office

Melbury House, 1-3 Oxford Road

Bournemouth University

Lansdowne Campus

BournemouthBH8 8ES.

\section{Authors' contributions}

$\mathrm{JO}, \mathrm{CC}, \mathrm{AH}, \mathrm{JB}$, and OA contributed to the development of the project, design of the study and drafted the manuscript. All authors read and approved the final manuscript.

\section{Ethics approval and consent to participate}

The study has been approved the UK Heath Research Authority, Bournemouth University ethics panel, and R\&D at Salford Royal NHS Foundation Trust (IRAS 228901). The declaration of Helsinki will be adhered to during this study. All data will be anonymised and participants must provide written informed consent. Insurance is provided through Bournemouth University. The procedure for withdrawal from the study is in line with the HRA non CITMP study protocol. Severe adverse events reported to the $\mathrm{Cl}$ or the neurologists and will result in their withdrawal and appropriate referral to their General practitioner or neurologist. The study may be monitored in accordance with SRFT'S R \&D department. Standard operating procedures, to ensure compliance with GCP and the Research Governance Framework 2005. All trial related documents will be made available upon request for monitoring by $R \& D$ monitors. Any changes to the protocol will be communicated as per the HRA guidelines.

\section{Consent for publication}

Not applicable

\section{Competing interests}

The authors declare that they have no competing interests.

\section{Publisher's Note}

Springer Nature remains neutral with regard to jurisdictional claims in published maps and institutional affiliations.

\section{Author details}

${ }^{1}$ Bournemouth University, Royal London House, Christchurch Road, Bournemouth BH1 3LT, UK. ' BPP University, McTimoney College, Kimber Road, Abingdon OX14 1BZ, UK.

Received: 21 August 2018 Accepted: 3 January 2019

Published online: 27 March 2019

\section{References}

1. Zebenholzer K, Andree C, Lechner A, Broessner G, Lampl C, Luthringshausen G, Wuschitz A, Obmann S, Berek K, Wöber C. Prevalence, management and burden of episodic and chronic headaches - a cross sectional multicentre study in eight Austrian headache centres. J Headache Pain. 2015;16(1):1-9.

2. Messali A, Sanderson J, Blumenfeld A, Goadsby P, Buse D, Varon S, Stokes M, Lipton R. Direct and indirect costs of chronic and episodic migraine in the United States: a web based survey. Headache: The Journal of Head and Face Pain. 2016:56(2):306-22.

3. Galego J, Cipullo J, Cordeiro J, Tognola W. Clinical features of episodic migraine and transformed migraine: a comparative study. Arquivos de NeuroPsiquiatria. 2002;60(4):912-6.

4. Anon. ICHD-3 The International Classification of Headache Disorders 3rd edition [Internet]. ICHD-3 The International Classification of Headache Disorders 3rd edition. 2018: https://www.ichd-3.org. Accessed 30 July 2018.

5. McCrone P, Seed P, Dowson A, Clark L, Goldstein L, Morgan M, Ridsdale L. Service use and costs for people with headache: a UK primary care study. J Headache Pain. 2011;12(6):617-23.

6. Bloudek L, Stokes M, Buse D, Wilcox T, Lipton R, Goadsby P, Varon S, Blumenfeld A, Katsarava Z, Pascual J, Lanteri-Minet M, Cortelli P, Martelletti P. Cost of healthcare for patients with migraine in five European countries: results from the international burden of migraine study (IBMS). J Headache Pain. 2012; 13(5):361-78.

7. Berra E, Sances G, De Icco R, Avenali M, Berlangieri M, De Paoli I, Bolla M, Allena M, Ghiotto N, Guaschino E, Cristina S, Tassorelli C, Sandrini G, Nappi G. Cost of chronic and episodic migraine: a pilot study from a tertiary headache Centre in northern Italy. J Headache Pain. 2015;16(1):1-8.

8. Lanteri-Minet M. Economic burden and costs of chronic migraine. Curr Pain Headache Rep. 2013;18(1):385-95.

9. Katsarava Z, Buse D, Manack A, Lipton R. Defining the differences between episodic migraine and chronic migraine. Curr Pain Headache Rep. 2013; 16(1):86-92 
10. Natoli J, Manack A, Dean B, Butler Q, Turkel C, Stovner L, Lipton R. Global prevalence of chronic migraine: a systematic review. Cephalalgia. 2009;30(5): 599-609.

11. Finocchi C, Strada L. Sex-related differences in migraine. Neurol Sci. 2014; 35(S1):207-13.

12. Steiner T, Stovner L, Vos T. GBD 2015: migraine is the third cause of disability in under 50s. J Headache Pain. 2016;17(1):1-4.

13. Buse D, Loder E, Gorman J, Stewart W, Reed M, Fanning K, Serrano D, Lipton R. Sex differences in the prevalence, symptoms, and associated features of migraine, probable migraine and other severe headache: results of the American migraine prevalence and prevention (AMPP) study. Headache: The Journal of Head and Face Pain. 2013;53(8):1278-99.

14. Blumenfeld A, Varon S, Wilcox T, Buse D, Kawata A, Manack A, Goadsby P, Lipton R. Disability, HRQoL and resource use among chronic and episodic migraineurs: results from the international burden of migraine study (IBMS). Cephalalgia. 2010;31(3):301-15.

15. Gaul C, van Doorn C, Webering N, Dlugaj M, Katsarava Z, Diener H, Fritsche G Clinical outcome of a headache specific multidisciplinary treatment program and adherence to treatment recommendations in a tertiary headache center: an observational study. J Headache Pain. 2011;12(4):475-83.

16. Diener H, Solbach K, Holle D, Gaul C. Integrated care for chronic migraine patients: epidemiology, burden, diagnosis and treatment options. Clin Med. 2015;15(4):344-50.

17. Blumenfeld A, Gennings C, Cady R. Pharmacological synergy: the next frontier on therapeutic advancement for migraine. Headache: The Journal of Head and Face Pain. 2012:52(4):636-47.

18. Silberstein S. Topiramate in migraine prevention: a 2016 perspective Headache: The Journal of Head and Face Pain. 2016;57(1):165-78.

19. Anon. Headaches in over 12 s: diagnosis and management / Guidance and guidelines | NICE [online]. Nice.org.uk. 2012; https://www.nice.org.uk/gui dance/cg150 [Accessed 30 Mar 2018].

20. Khalil M, Zafar H, Quarshie V, Ahmed F. Prospective analysis of the use of OnabotulinumtoxinA (BOTOX) in the treatment of chronic migraine; real-life data in 254 patients from Hull, UK. J Headache Pain. 2014;15(1):1-9.

21. Gooriah R, Ahmed F. OnabotulinumtoxinA for chronic migraine: critical appraisal. Ther Clin Risk Manag. 2015;11:1003-13.

22. Mathew N, Jaffri S. A double- blind comparison of OnabotulinumtoxinA (BOTOX ${ }^{\circledR}$ ) and Topiramate (TOPAMAX ${ }^{\circledR}$ ) for the prophylactic treatment of chronic migraine: a pilot study. Headache: The Journal of Head and Face Pain. 2009:49(10):1466-78.

23. Chiang $C$, Starling A. OnabotulinumtoxinA in the treatment of patients with chronic migraine: clinical evidence and experience. Ther Adv Neurol Disord. 2017;10(12):397-406

24. Frampton J. OnabotulinumtoxinA (BOTOX ${ }^{\circledR}$ ). Drugs. 2012;72(6):825-45.

25. Cady R, Schreiber $C$. Botulinum toxin type a as migraine preventive treatment in patients previously failing oral prophylactic treatment due to compliance issues. Headache: The Journal of Head and Face Pain. 2008:48(6):900-13.

26. Chaibi A, Russell M. Manual therapies for primary chronic headaches: a systematic review of randomized controlled trials. J Headache Pain. 2014; 15(1):1-8.

27. Clar C, Tsertsvadze A, Court R, Hundt G, Clarke A, Sutcliffe P. Clinical effectiveness of manual therapy for the management of musculoskeletal and non-musculoskeletal conditions: systematic review and update of UK evidence report. Chiropr Manual Therap. 2014;22(1):12.

28. Buture A, Gooriah R, Nimeri R, Ahmed F. Current understanding on pain mechanism in migraine and cluster headache. Anesthesiol Pain Med. 2016 6(3):1-8.

29. Coppola G, Di Lorenzo C, Schoenen J, Pierelli F. Habituation and sensitization in primary headaches. J Headache and Pain. 2013;14(1):65.

30. Dodick D, Silberstein S. Central sensitization theory of migraine: clinica complications. Headache: The Journal of Head and Face Pain. 2006;46(s4): 182-91.

31. Borsook D, Maleki N, Becerra L, McEwen B. Understanding migraine through the Lens of maladaptive stress responses: a model disease of allostatic load. Neuron. 2012;73(2):219-34.

32. Cortelli P, Pierangeli G, Montagna P. Is migraine a disease? Neurol Sci. 2010; 31(S1):29-31.

33. Burstein $\mathrm{R}$, Noseda $\mathrm{R}$, Borsook D. Migraine: multiple processes, complex pathophysiology. J Neurosci. 2015;35(17):6619-29.

34. Diener $\mathrm{H}$, Holle D, Dodick D. Treatment of chronic migraine. Current Pain Headache Reports. 2010;15(1):64-9.
35. Negro A, Martelletti P. Chronic migraine plus medication overuse headache: two entities or not? J Headache Pain. 2011;12(6):593-601.

36. Rojo E, Pedraza M, Muñoz I, Mulero P, Ruiz M, de la Cruz C, et al. Chronic migraine with and without medication overuse: experience in a hospital series of 434 patients. Neurología (English Edition). 2015;30(3):153-7.

37. Bigal M, Lipton R. Migraine Chronification. Curr Neurol Neurosci Rep. 2011; 11(2):139-48.

38. Benatto M, Florencio L, Carvalho G, Dach F, Bigal M, Chaves T, BevilaquaGrossi D. Cutaneous allodynia is more frequent in chronic migraine, and its presence and severity seems to be more associated with the duration of the disease. Arquivos de Neuro Psiquiatria. 2017;75(3):153-9.

39. Zappaterra M, Guerzoni S, Cainazzo M, Ferrari A, Pini L. Basal cutaneous pain threshold in headache patients. J Headache Pain. 2017;12(3):303-10.

40. Adams A, Serrano D, Buse D, Reed M, Marske V, Fanning K, Lipton R. The impact of chronic migraine: the chronic migraine epidemiology and outcomes (CaMEO) study methods and baseline results. Cephalalgia. 2014:35(7):563-78.

41. Mathew P, Cutrer F, Garza I. A touchy subject: an assessment of cutaneous allodynia in a chronic migraine population. J Pain Res. 2016;9:101-4.

42. Nijs J, Meeus M, Van Oosterwijck J, Roussel N, De Kooning M, Ickmans K, Matic M. Treatment of central sensitization in patients with 'unexplained' chronic pain: what options do we have? Expert Opin Pharmacother. 2011;12(7):1087-98.

43. Bialosky J, Bishop M, Price D, Robinson M, George S. The mechanisms of manual therapy in the treatment of musculoskeletal pain: a comprehensive model. Man Ther. 2009;14(5):531-8.

44. Bishop M, Torres-Cueco R, Gay C, Lluch-Girbés E, Beneciuk J, Bialosky J. What effect can manual therapy have on a patient's pain experience? Pain Management. 2015;5(6):455-64

45. Nijs J, Van Houdenhove B, Oostendorp R. Recognition of central sensitization in patients with musculoskeletal pain: application of pain neurophysiology in manual therapy practice. Man Ther. 2010;15(2):135-41.

46. Bryans R, Descarreaux M, Duranleau M, Marcoux H, Potter B, Ruegg R, Shaw L, Watkin R, White E. Evidence based guidelines for the chiropractic treatment of adults with headache. J Manip Physiol Ther. 2011;34(5):274-89.

47. Fernández-de-las-Peñas C, Dommerholt J. Myofascial trigger points: peripheral or central phenomenon? Curr Rheumatol Rep. 2013;16(1):395-401.

48. Miller J, Gross A, D'Sylva J, Burnie S, Goldsmith C, Graham N, Haines T, Bronfort G, Hoving J. Manual therapy and exercise for neck pain: a systematic review. Man Ther. 2010;15(4):334-54.

49. Bevilaqua-Grossi D, Gonsalves M, Carvalho G, Florencio L, Dach F, Speciali J, Bigal M, Chaves T. Additional effects of a physical therapy protocol on headache frequency, pressure pain threshold, and improvement perception in patients with migraine and associated neck pain: a randomized controlled trial. Arch Phys Med Rehabil. 2016;97(6):866-74.

50. Grossi D, Chaves T, Gonçalves M, Moreira V, Canonica A, Florencio L, Bordini C, Speciali J, Bigal M. Pressure pain threshold in the craniocervical muscles of women with episodic and chronic migraine: a controlled study. Arquivos de Neuro Psiquiatria. 2011;69(4):607-12

51. Diener H, Dodick D, Goadsby P, Lipton R, Olesen J, Silberstein S. Chronic migraine_classification, characteristics and treatment. Nat Rev Neurol. 2012; 8(3):162-71.

52. Silberstein S, Tfelt-Hansen P, Dodick D, Limmroth V, Lipton R, Pascual J, Wang S. Guidelines for controlled trials of prophylactic treatment of chronic migraine in adults. Cephalalgia. 2008;28(5):484-95.

53. Boutron I, Altman DG, Moher D, Schulz KF, Ravaud P, DJ C, et al. CONSORT Statement for Randomized Trials of Non-pharmacologic Treatments: A 2017 Update and a CONSORT Extension for Non-pharmacologic Trial Abstracts. Ann Intern Med. Am Coll Physicians. 2017;167(1):40.

54. Yang M, Rendas-Baum R, Varon S, Kosinski M. Validation of the headache impact test (HIT-6 ${ }^{\mathrm{TM}}$ ) across episodic and chronic igraine. Cephalalgia. 2010; 31(3):357-67.

55. Suh G, Park J, Shin H. Differences in clinical features and disability according to the frequency of medication use in patients with chronic migraine. J Clin Neurol. 2012:8(3):198.

56. Aurora S, Dodick D, Turke C, DeGryse R, Silberstein S, Lipton R, Diener H, Brin M. OnabotulinumtoxinA for treatment of chronic migraine: results from the double-blind, randomized, placebo-controlled phase of the PREEMPT 1 trial. Cephalalgia. 2010;30(7):793-803.

57. Rendas-Baum R, Yang M, Varon S, Bloudek L, DeGryse R, Kosinski M. Validation of the headache impact test (HIT-6) in patients with chronic migraine. Health Qual Life Outcomes. 2014;12(1):110-27. 
58. Negro A, Curto M, Lionetto L, Crialesi D, Martelletti P. OnabotulinumtoxinA $155 \mathrm{U}$ in medication overuse headache: a two years prospective study. SpringerPlus. 2015:4(1):826-34.

59. Rendas-Baum R, Bloudek L, Maglinte G, Varon S. The psychometric properties of the migraine specific quality of life questionnaire version 2.1 (MSQ) in chronic migraine patients. Qual Life Res. 2012;22(5):1123-33.

60. Urbaniak G C, Plous S. (2013). Research randomizer (version 4.0) [computer software]. Retrieved on June 22, 2013, from http://www.randomizer.org/

61. MacPherson H, Newbronner E, Chamberlain R, Hopton A. Patients' experiences and expectations of chiropractic care: a national cross-sectional survey. Chiropr Manual Therap. 2015;23(1):3.

62. AB S. SomedicSenseLab AB Algometer, 2018 [online]. http://somedic.com/ en/algometer.html [Accessed 21 Jun 2018].

63. Marteau T, Bekker $\mathrm{H}$. The development of a six item short form of the state scale of the Spielberger StateTrait anxiety inventory (STAI). Br J Clin Psychol. 1992;31(3):301-6.

64. Cerritelli F, Ginevri L, Messi G, Caprari E, Di Vincenzo M, Renzetti C, Cozzolino V, Barlafante G, Foschi N, Provinciali L. Clinical effectiveness of osteopathic treatment in chronic migraine: 3-armed randomized controlled trial. Complement Therap Med. 2015;23(2):149-56.

65. Chaibi A, Benth J, Tuchin P, Russell M. Chiropractic spinal manipulative therapy for migraine: a three-armed, single-blinded, placebo, randomized controlled trial. Eur J Neurol. 2016;24(1):143-53.

66. Gandolfi M, Marchioretto F, Turrina A, Dimitrova E, Geroin C, Valè N. Does myofascial and trigger point treatment reduce pain and analgesic intake in patients undergoing OnabotulinumtoxinA injection due to chronic intractable migraine? A pilot, single-blind randomized controlled trial. European J Physical Rehabilit Med. 2017:54(1):1-12.

67. Möller HJ. Effectiveness studies: advantages and disadvantages. Dialogues Clin Neurosci. 2011;13:199-207.

68. Blanchard E, Andrasik F, Neff D, Jurish S, O'Keefe D. Social validation of the headache diary. Behav Ther. 1981;12(5):711-5.

69. Peng K, Wang S. Migraine diagnosis: Screening items, instruments, and scales. ActaAnaesthesiologicaTaiwanica. 2010;50(2):69-73.

70. Jensen R, Tassorelli C, Rossi P, Allena M, Osipova V, Steiner T, Sandrini G, Olesen J, Nappi G, Barrientos N, Mulleners W, Dzagnidze A, Figuerola M, Filatova E, Katsarava Z, Koreshkina M, Milanov I, Pereira-Monteiro J, Perrotta A, Ratchin A, Radojicic A, Rezzani C, Sances G, Sretenovic S, Torelli P. A basic diagnostic headache diary $(B D H D)$ is well accepted and useful in the diagnosis of headache. A multicentre European and Latin American study. Cephalalgia. 2011; 31(15):1549-60

71. Meissner K, Fässler M, Rücker G, Kleijnen J, Hróbjartsson A, Schneider A, Antes G, Linde K. Differential effectiveness of placebo treatments. JAMA Intern Med. 2013:173(21):1941.

72. Diener H, Charles A, Goadsby P, Holle D. New therapeutic approaches for the prevention and treatment of migraine. The Lancet Neurology. 2015; 14(10):1010-22.

73. Speciali J, Peres M, Bigal M. Migraine treatment and placebo effect. Expert Rev Neurother. 2010;10(3):413-9.

74. Schwedt T, Hentz J, Dodick D. Factors associated with the prophylactic effect of placebo injections in subjects enrolled in a study of botulinum toxin for migraine. Cephalalgia. 2007;27(6):528-34.

75. Diener $H$, Schorn C, Bingel U, Dodick D. The importance of placebo in headache research. Cephalalgia. 2008;28(10):1003-11.

76. Benedetti F. Placebo effects: from the neurobiological paradigm to translational implications. Neuron. 2014:84(3):623-37.

77. Fortin M, Smith S. Improving the external validity of clinical trials: the case of multiple chronic conditions. J Comorbidity. 2013;3:30-5.

78. Hougaard A, Tfelt-Hansen P. General lack of use of placebo in prophylactic, randomised, controlled trials in adult migraine. A systematic review. Cephalalgia. 2016;36(10):960-9.

79. Schwedt T. Chronic migraine. BMJ. 2014;348:1416.

80. Cohen S, Kamarck T, Mermelstein R. A global measure of perceived stress. J Health Soc Behav. 1983:24(4):385

81. Carver C. You want to measure coping but your protocol' too long: consider the brief cope. Int J Behav Med. 1997:4(1):92-100.

82. Zigmond A, Snaith R. The hospital anxiety and depression scale. Acta Psychiatr Scand. 1983;67(6):361-70.
83. Lipton R, Bigal M, Ashina S, Burstein R, Silberstein S, Reed M, Serrano D, Stewart W. Cutaneous allodynia in the migraine population. Ann Neurol. 2008;63(2):148-58.

84. Neziril A, Scaramozzinol P, Andersenl O, Dickensonl A, Arendt-Nielsenl L, Curatolol M. Reference values of mechanical and thermal pain tests in a pain free population. Eur J Pain. 2011;15(4):376-83.

85. Hurst $\mathrm{H}$, Bolton J. Assessing the clinical significance of change scores recorded on subjective outcome measures. J Manip Physiol Ther. 2004;27(1):26-35.

\section{Ready to submit your research? Choose BMC and benefit from:}

- fast, convenient online submission

- thorough peer review by experienced researchers in your field

- rapid publication on acceptance

- support for research data, including large and complex data types

- gold Open Access which fosters wider collaboration and increased citations

- maximum visibility for your research: over $100 \mathrm{M}$ website views per year

At BMC, research is always in progress.

Learn more biomedcentral.com/submissions 